\title{
Multifunctional proline rich proteins and their role in regulating cellular proline content in plants under stress
}

\author{
R.S. GUJJAR ${ }^{1 *}$, A.D. PATHAK ${ }^{1}$, S.G. KARKUTE ${ }^{2}$, and K. SUPAIBULWATANA ${ }^{3}$ \\ Division of Crop Improvement, Indian Institute of Sugarcane Research, Lucknow, 226002, Indial \\ Division of Crop Improvement, Indian Institute of Vegetable Research, Varanasi, 221305, India ${ }^{2}$ \\ Department of Biotechnology, Faculty of Science, Mahidol University, Bangkok, 10400, Thailand ${ }^{3}$
}

\begin{abstract}
Proline rich proteins (PRPs), earlier famous as animal salivary proteins, have now been proven as indispensable plant proteins. They are highly rich in proline amino acid residues at the N-terminus whereas a characteristic eight cysteine motif is located at the C-terminus. The PRPs support a number of developmental processes from germination to plant death. Under normal environmental conditions, $P R P$ genes express customarily in different plant parts depending on the specific function to be carried out. During abiotic stresses, $P R P$ genes exhibit an uneven pattern of transcriptional regulation depending on the time and intensity of stress. Transgenic plants overexpressing $P R P$ genes show an enhanced tolerance to abiotic stresses. This review focuses on contemporary functions of PRPs during stresses and proposes that PRPs are involved in the regulation of free cellular proline content during stress in a well synchronized manner.
\end{abstract}

Additional key words: abiotic and biotic stresses, PRP genes, transgenic plants

\section{Introduction}

Proline rich proteins (PRPs) were initially identified as salivary proteins of mammals. During 1960s, PRPs were reported in plants when hydroxyproline rich proteins were obtained by enzymatic degradation of cell wall (Lamport 1969). Later, it was confirmed that hydroxyproline rich proteins are derived by hydroxylation of PRPs on rough endoplasmic reticulum in the cytoplasm and transported to cell wall (Ridge and Osborne 1970, Pitzschke et al. 2016). The PRPs, having tandem repeats of a hexapeptide PPPVHL, were first identified and characterized in maize endosperm (Esen et al. 1982). Three proline rich proteins of soybean (SbPRP1, SbPRP2, and SbPRP3) were extensively studied for their function, localization, and differential expression (Hong et al. 1989, 1990, Francisco and Tierney 1990, Wyatt et al. 1992). The PRPs consist of two domains, a proline rich domain at the N-terminus (Dvorakova et al. 2007) and a typical hydrophobic eight cysteine motif (8-CM) at the C-terminus (Jose-Estanyol et al. 2004). The 8-CMC-terminal domain is a characteristic feature of lipid transfer protein (LTP) family/protease inhibitor/hydrophobic seed storage proteins (Jose-Estanyol et al. 2004), but a classical LTP is devoid of proline rich domain. The classification of PRPs is mainly based on the sequence and distribution of proline repeat motifs throughout the protein. Two broad classes of proline rich proteins are: simple PRPs that are devoid of a hydrophobic domain, and hybrid PRPs having a hydrophobic domain at the $\mathrm{C}$ terminus.

Preliminary research on plant proline/hydroxyproline rich proteins suggests their localization on cell wall (Lamport 1969, Deutch et al. 1995, Fowler et al. 1999) but recent reports show the subcellular localization of plant PRPs/hybrid PRPs (HyPRPs) at plasmalemma (Zhan et al. 2012, Qin et al. 2013, Li et al. 2014). The PRPs have

Submitted 4 July 2018, last revision 12 December 2018, accepted 15 January 2019.

Abbreviations: 8-CM - eight cysteine motif; LTP - lipid transfer protein; P5CS - pyrroline-5-carboxylate synthase; PRP - proline rich protein;

Acknowledgements: We thank Dr. M. Singh and Dr. H.C. Prasanna for providing useful suggestions and support. We are thankful to the colleagues for their work on this topic to provide literature for this review. The authors apologize to those whose work could not be cited. * Corresponding author; present address: Department of Biotechnology, Faculty of Science, Mahidol University, Bangkok, Thailand, e-mail: ranjit.gujjar@icar.gov.in 
been reported to play a crucial role in plant growth and developmental processes particularly during germination and flower development. Investigation during the past decade has confirmed that the expression of PRPs is modulated by biotic and abiotic stresses like chilling, water deficit, salinity, abscisic acid or salicylic acid treatments, and a viral inoculation. A specific localization of PRPs in different plant organs expedites the variability in their functions. This review focuses on a meticulous analysis of the functionality and molecular diversity of these multifunctional proteins. Further, participation of PRPs in both biotic and abiotic stresses has been discussed. There are two interesting facts that encouraged us to suggest a link between PRPs and free cellular proline. Firstly, as the name suggests, PRPs have a proline rich domain consisting of about $70 \%$ of proline residues. Secondly, PRPs demonstrate irregular patterns of expression under stress conditions.

\section{Sequence diversity in PRPs and their similarity with lipid transfer proteins}

Both PRPs and LTPs belong to a common superfamily of proteins called prolamins (Edstam et al. 2011). An $\mathrm{N}$-terminal proline rich domain (Fig. 1 Suppl.) and C-terminal 8-CM (Fig. 2 Suppl.) domains were aligned separately for the assessment of sequence diversity. Proline rich domains of PRPs portray different patterns of tandem repeats: PPVV, PPIV, PPYV, etc. Few PRPs have a continuous stretch of three or four proline residues: PPPIV, PPPYV, PPPVV, PPPS, PPPPIV, PPPPYV, and PPPPTP. Conversely, a C-terminal 8-CM domain is highly conserved in PRPs. The common feature of this domain is the presence of eight cysteine amino acid residues at specified positions and the abundance of leucine residues all over the stretch (Jose-Estanyol et al. 2004). There is a notable difference in number of leucine residues between the 8-CM domain of PRPs (12 - 13 L) and LTPs (9 L). Motif search along PRP sequences exposes the presence of three key motifs: a hydrophobic seed protein, probable LTP 2 , and tryptophan $\alpha$-amylase. Besides, some PRPs have histone H1-like nucleoprotein $\mathrm{HC} 2$, proteasome subunit, proteins of unknown function (DUF1409), a transposaseassociated domain, and the SOCE-associated regulatory factor of calcium homoeostasis.

Though the 8-CM domain is functionally analogous in both PRPs and a classical LTP family (Dvorakova et al. 2007), there are many notable differences in the sequence. The 8-CM domain of PRPs has an average stretch of 82 amino acid residues from the cysteine residue placed at the first position to the cysteine residue at the eighth position compared to classical LTPs, which have 79 amino acid residues along this stretch (Fig. 3 Suppl.). Differences in structure between the 8-CM domain of PRP and LTP have been analysed by comparing their 3D structures generated by the $i$-TASSAR server (Yang et al. 2015). The 8-CM domains of both the proteins consist of four $\alpha$-helices interconnected with three loops, representing a typical membrane protein structure. The fourth helix of LTPs is comparatively more extended than that of PRP. Comparison of 3D structures between the 8-CM domain of PRP and LTP is represented in Fig. 4 Suppl. Despite the significant difference in amino acid residues between both the domains, their 3D structure is almost the same, portraying the conservation of structure for carrying out the same functions at the cellular level.

\section{Developmental functions of PRPs}

The PRPs are key players in plant development from germination of seeds to morphogenesis of different organs and ultimately to cell death. Though there are many other factors inducing germination of seeds, PRPs have been identified as important regulators of germination in soybean (Hong et al. 1989), Medicago truncatula (Bouton et al. 2005), cotton (Qin et al. 2013), tobacco (Chen et al. 2014), rice (Mellacheruvu et al. 2016), and many more plants. During the course of germination, their expression is high in the apical region of hypocotyl (Hong et al. 1989) and embryo axis (Bouton et al. 2005). During the reproductive stage, PRPs are preferentially expressed in floral tissues (petals, pollen grains, pollen tubes, ovules, and zygotes) where they promote flower development and pollen tube growth (Gothandam et al. 2010, Qin et al. 2013, Chen et al. 2014). Under normal conditions, PRPs are constitutively expressed in roots and vegetative tissues (Bouton et al. 2005, Peng et al. 2015). The PRPL1 gene of Arabidopsis is peculiarly expressed in root hairs and triggers their elongation (Boron et al. 2014). Involvement of PRPs in cell elongation is further supported by overexpression of the HyPRP gene in tobacco BY-2 cell lines where calli size increases in suspension culture as well as on solid media (Dvorakova et al. 2012). The process of cell elongation may be due to loosening cell wall triggered by the hydrophobic 8-CM domain of PRPs. The hydrophobic $8 \mathrm{CM}$ domain of LTPs has been reported to interrupt hydrogen bonds between cellulose and hemicelluloses leading to non-hydrolytic loosening these cross-links (Nieuwland et al. 2005). Furthermore, a novel observation came out in Arabidopsis where a SICKLE protein (PRP) was found to be involved in microRNA biogenesis and degradation of spliced introns. SICKLE loss of function mutants exhibit pleiotropic developmental defects, such as reduction in plant height, delay in flowering, increase in serration at the leaf margin, and abnormal inflorescence phyllotaxy (Zhan et al. 2012). Recently, a hybrid PRP of Glycine soja (GsEARLI17) has been reported to influence cuticle formation as thicker cuticle asere observed in transgenic lines of Arabidopsis over-expressing this gene (Liu et al. 2015).

\section{Puzzling expression of PRPs during abiotic stresses}

Abiotic stresses modulate transcription of $P R P$ genes 
remarkably. Some investigations support their downregulation whereas other favour up-regulation in different plant species. Furthermore, a notable number of investigations demonstrated a temporal regulation of PRP genes depending on the period and intensity of abiotic stress. A microarray experiment performed in our laboratory showed a significant down-regulation of $P R P$ gene expression in tomato under osmotic stress. Reverse transcription quantitative PCR analysis revealed downregulation of an S1PRP gene under drought stress in all tomato tissues: root (1314-fold), stem (11-fold), leaf (40-fold), and flower (2-fold) (Gujjar et al. 2014, 2018). Similar results were reported earlier in Solanum tuberosum (Menke et al. 2000) and in Poncirus trifoliate (Peng et al. 2015) where osmotic stress lead to gradual decrease in transcription of $P R P$ genes.

On the other hand, contrasting observations favouring up-regulation of $P R P$ genes at various abiotic stresses were reported in Phaseolus vulgaris (Battaglia et al. 2007), cotton (Qin et al. 2013), and Glycine soja (Liu et al. 2015). Temporal and spatial variations in expression of PRP genes were also reported in soybean (He et al. 2002) and Poncirus trifoliate (Peng et al. 2015). The expression of an $S b P R P$ gene in soybean is specifically limited to leaf and epicotyle in response to multiple stresses. Upon exposure to salt stress, the $S b P R P$ gene is up-regulated initially at $0.1 \% \mathrm{NaCl}$ reaching a maximum up-regulation at $0.4 \% \mathrm{NaCl}$; then the expression decreases (He et al. 2002). No SbPRP transcription is detected at $2 \% \mathrm{NaCl}$. It seems that expression of $P R P$ genes is modulated under stress according to the requirement of a plant. In either way, PRPs project themselves as beneficial proteins under all types of environmental stresses. It has been confirmed by their over-expression and silencing in transgenic plants where over-expression of $P R P$ genes undisputedly results in enhanced tolerance to multiple abiotic stresses (Barthakur et al. 2001, Gothandam et al. 2010, Priyanka et al. 2010, Liu et al. 2015, Mellacheruvu et al. 2016). Recently, promotor analysis of PRP genes in Nicotiana tabaccum and Glycine soja evidenced the presence of stress inducible elements, viz., W-boxes (WRKY-binding sites), a GATA box (the binding site for basic helix-loophelix), and MYBs on it (Chen et al. 2014, Liu et al. 2015).

\section{Proline rich proteins during biotic stress}

Though implications of PRPs in biotic stress tolerance are limited to only few reports, their contribution has been well documented in providing tolerance against viral (He et al. 2002), bacterial (Li et al. 2014, Cecchini et al. 2015), and fungal (Mellacheruvu et al. 2016) pathogens. Treatments with salicylic acid and methyl jasmonate trigger the accumulation of PRPs in soybean (He et al. 2002) and Arabidopsis (Li et al. 2014). The soybean mosaic virus induces $S b P R P$ expression during initial stages of inoculation, but its expression decreases thereafter (He et al. 2002). Treatments with virulent and avirulent strains also induce expression of a double hybrid PRP AtDHyPRPl in Arabidopsis (Li et al. 2014). Further, transgenic lines of Arabidopsis over-expressing the AtDHyPRP1 gene exhibit an improved tolerance to bacterial suspension of virulent PstDC3000 whereas silencing AtDHyPRP1 by RNA interference confers susceptibility of plants to virulent PstDC3000. (Li et al. 2014). Transgenic rice plants overexpressing a hybrid PRP from Cajanus cajan (CcHyPRP) exhibited enhanced resistance to a fungal pathogen Magnaporthe grisea, which causes blast disease (Mellacheruvu et al. 2016). However, research on Capsicum annuum and Nicotiana benthamiana showed PRPs as positive regulators of cell death. This is evidenced by pathogen induced cell death in Nicotiana benthamiana transgenic plants over-expressing an HyPRP1 gene (Yeom et al. 2012). It was also suggested that cell death in these transgenic plants may be due to the down-regulation of reactive oxygen species scavenging genes and an enhanced pathogen susceptibility. Moreover, silencing the HyPRP1 gene suppresses pathogen induced cell death and enhances disease resistance in tobacco signifying the role of $H y P R P 1$ as a negative regulator of basal defence against pathogens (Yeom et al. 2012).

\section{Proline rich proteins and proline content during stress: is there any link?}

Proline is an excellent osmolyte that protects subcellular structures and macromolecules under osmotic stress (Szabados and Savoure 2010, Meringer et al. 2016). Some of the core functions of proline during stress include reactive oxygen species scavenging activity during oxidative stress (Smirnoff and Cumbes 1989, Matysik et al. 2002), protection of nitrate reductase, ribonucleases, and proteases during heavy metal stress (Sharma and Dubey 2005, Mishra and Dubey 2006), and molecular chaperoning to protect integrity of proteins and enzymes during extreme temperatures (Rajendrakumar et al. 1994). After experiencing stress, oxidation of proline by proline dehydrogenase and P5C dehydrogenase provides electrons for mitochondrial respiratory chain and contributes to energy supply for resumed growth (Hare and Cress 1997, Kishor et al. 2005). Though proline has plenty of protective functions, its high content ( $40-50 \mathrm{mM})$ may inhibit growth and cell division in plants (Maggio et al. 2002, Hayat et al. 2012). Excess proline has been reported to inhibit seed germination (Hare et al. 2003), restrict growth of plants (Yamada et al. 2005), and arrest root growth (Kant et al. 2006). It may cause a feedback inhibition of pyrroline5-carboxylate synthase (P5CS) which, in turn, blocks proline biosynthesis and affects the NADP+/NADPH ratio and redox balance in plastids leading to chlorophyll damage (Mani et al. 2002, Nanjo et al. 2003). Interestingly, transcript profiling in Arabidopsis revealed that about one third of rehydration-inducible genes are triggered by a high proline content (Oono et al. 2003). Thus, proline may be considered as an undisputed indicator of all types of stresses, and regulation of its content in plants is of an utter importance. 
Till date, a well demonstrated process for proline accumulation in plants during stress is its synthesis through P5CS (Kishor et al. 1995, Perez-Arellano et al. 2010). Some results disapprove a hypothesis that proline accumulation is solely dependent on P5CS. The earliest evidence that an increase in free proline content during water stress is due to cell wall bounded protein degradation was given by Chen et al. (1964). The amount of free proline which accumulates during stress was quantified in the following years, and was found to be considerably higher than the content of bounded protein indicating de-novo synthesis of proline (Barnett and Naylor 1966, Thompson et al. 1966). In developing grapevine fruits, proline accumulation is independent and not associated with either an increase in amount of P5CS mRNA and proteins or a decrease in content of proline dehydrogenase (Stines et al. 1999). Furthermore, proline content does not correlate with a high amount of P5CS transcripts in roots of $A$. thaliana (Verbruggen and Hermans 2008). Alternatively, it was suggested that degradation of cell wall bound proteins and few PRPs may contribute to accumulation of proline during stress conditions (Chen et al. 1964, Barthakur et al. 2001).

Though the involvement of PRPs in various stresses has been well studied, their precise function in mitigating the stresses has not been investigated till date. However, increase or decrease in expressions of PRPs is largely advantageous to a plant in mitigating stress. Recently, we have found that transcription of a tomato SIPRP gene is reduced under drought stress in roots, stems, leaves, and flowers with the greatest reduction in roots. Simultaneously, proline content increases in all plant parts under drought stress with the highest increase in leaves (Gujjar et al. 2018). The interpretation may be that PRPs probably act as appendages to regulate cellular content of proline during and after the stress. Furthermore, it may be suggested that transcription of $P R P$ genes during stress is sagaciously synchronized to equilibrate cellular proline content as per cellular requirement.

\section{A suggested model for drought induced accumulation of proline}

On exposure to drought stress, proline accumulates in all plant tissues to maintain the osmotic balance. Content of proline in cells may increase by its de-novo synthesis by P5CS (Verslues and Sharma 2010, Hayat et al. 2012) and degradation of cell wall bound PRPs (Chen et al. 1964, Barthakur et al. 2001). Besides, down-regulation of PRP genes under drought stress (Gujjar et. al. 2014, Peng et al. 2015, Gujjar et al. 2018) indirectly helps in building up a high cellular proline content to counteract drought (Fig. 1A). The earliest upshot of drought stress is perceived by root cells where a high amount of P5CS transcripts is noticed, and de-novo synthesis of proline sets in motion

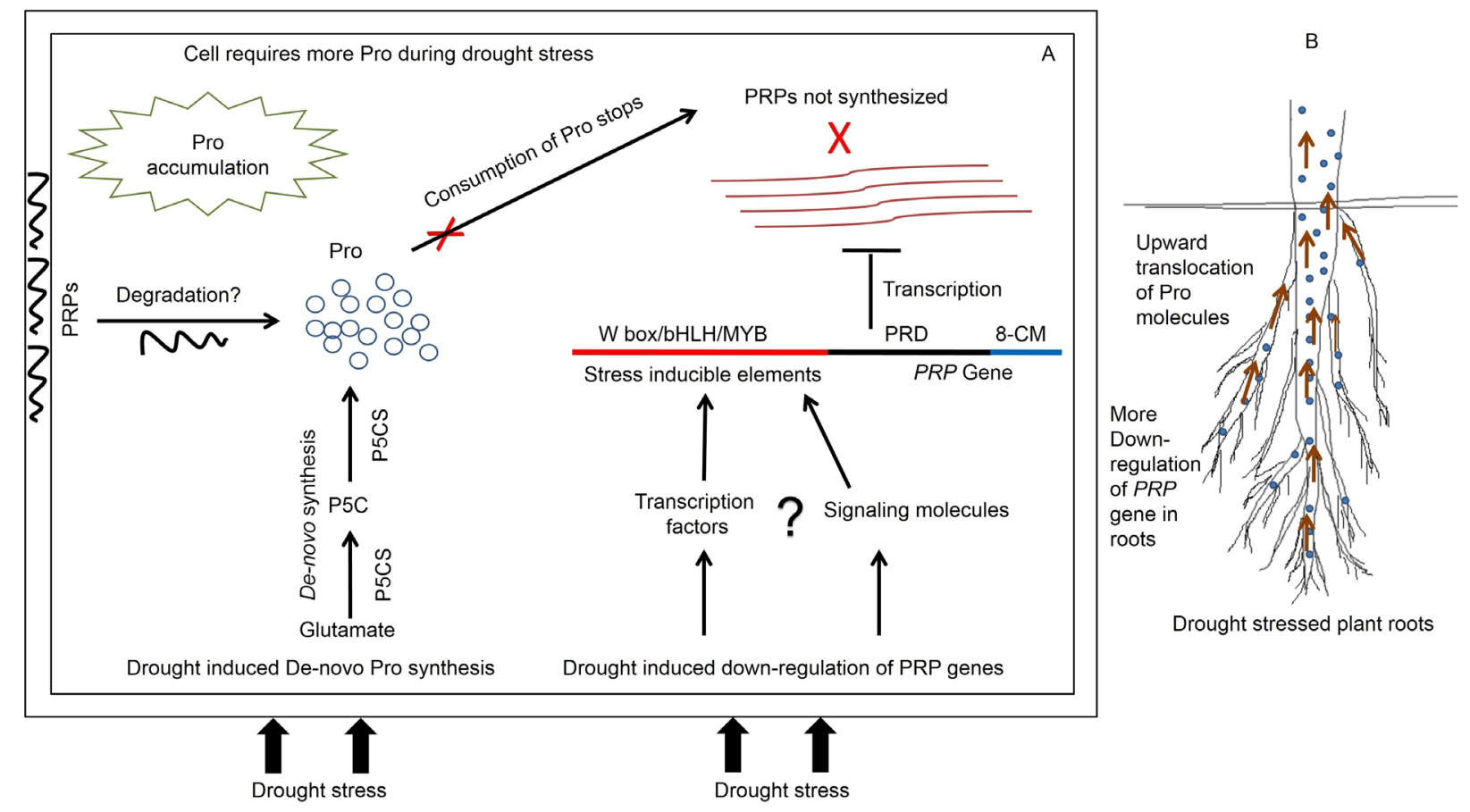

Fig. 1. A - Regulation of free cellular proline (Pro) content during drought stress in plants. Drought perception induces accumulation of Pro in plant cell by three ways: 1) de-novo synthesis of Pro mediated through pyrroline-5-carboxylate synthase (P5CS) pathway whereby glutamate is converted into Pro; 2) cell wall bound proline rich proteins (PRPs) are degraded to release free Pro inside the cell; 3) transcription of a PRP gene is repressed or blocked via some unknown signalling cascade. This results in a decreased synthesis of PRPs so as to avoid consumption of free cellular Pro. $B$ - Redistribution of proline from roots to shoots. Proline accumulated in roots during drought stress is translocated upwards through xylem to aerial plant parts resulting in a lesser content of Pro in roots (redrawn from Verbruggen and Hermans 2008). 
instantly. Moreover, the highest down-regulation of a $P R P$ gene in roots of tomato (Gujjar et al. 2018) also supports an enhanced synthesis of proline in roots. Interestingly, proline content of roots is relatively low and does not match with a high transcription of a P5CS gene in Arabidopsis roots (Hua et al. 1997, Verbruggen and Hermans 2008). This ambiguity may be attributed to the fact that proline is translocated upwards from roots to shoots (Fig. 1B) through xylem (Verbruggen and Hermans 2008). Besides this long distance translocation, intracellular as well as intercellular transport of proline is also evident during drought stress; it is carried out by specific proline transporters (Rentsch et al. 1996, Schwacke et al. 1999).

\section{Concluding remarks and future perspectives}

The PRPs have been studied under different environmental stresses where their expression is either increased, decreased, or transiently changed. Contemporary investigations suggest that the promoter region of $P R P$ genes has drought inducible elements, and these cis-acting elements serve as binding sites for WRKY transcription factors, basic helix-loop-helix proteins and MYBs (Chen et al. 2014, Liu et al. 2015). This finding may fascinate the curiosity of researchers in future to investigate more about

\section{References}

Barnett, N.M., Naylor, A.W.: Amino acid and protein metabolism in Bermuda grass during water stress. - Plant Physiol. 41: 1222-1230, 1966.

Barthakur, S., Babu, V., Bansal, K.C.: Over-expression of osmotin induces proline accumulation and confers tolerance to osmotic stress in transgenic tobacco. - J. Plant Biochem. Biotechnol. 10: 31-37, 2001.

Battaglia, M., Solorzano, R.M., Hernandez, M., Cuellar-Ortiz, S., Garcia-Gomez, B., Marquez, J., Covarrubias, A.A.: Prolinerich cell wall proteins accumulate in growing regions and phloem tissue in response to water deficit in common bean seedlings. - Planta 225: 1121-1133, 2007.

Boron, A.K., Orden, J.V., Markakis, M.N., Mouille, G., Adriaensen, D., Verbelen, J., Hofte, H., Vissenberg, K.: Proline-rich protein-like PRPL1 controls elongation of root hairs in Arabidopsis thaliana. - J. exp. Bot. 65: 5485-5495, 2014.

Bouton, S., Viau, L., Lelievre, E., Limami, A.M.: A gene encoding a protein with a proline-rich domain (MtPPRD1), revealed by suppressive subtractive hybridization (SSH), is specifically expressed in the Medicago truncatula embryo axis during germination. - J. exp. Bot. 56: 825-832, 2005.

Cecchini N.M., Steffes, K., Schläppi, M.R., Gifford, A.N., Greenberg, J.T.: Arabidopsis AZI1 family proteins mediate signal mobilization for systemic defence priming. - Nat. Commun. 23: 6, 2015.

Chen, D., Kessler, B., Monselise, S.P.: Studies on water regime and nitrogen metabolism of citrus seedlings grown under water stress. - Plant Physiol. 39: 379-386, 1964.

Chen, J., Zhao, J., Ning, J., Liu, Y., Xu, J., Tian, S., Zhang, L., Sun, M.X.: NtProRP1, a novel proline-rich protein, is an osmotic stress-responsive factor and specifically functions these proteins. It is obvious from literature that stress induces de novo synthesis of proline (the reaction catalysed by P5CS). Besides natural synthesis, two additional pathways may be suggested for proline accumulation in cells during exposure to abiotic stresses: 1) degradation of PRPs during osmotic stress to liberate free proline (Chen et al. 1964, Barthakur et al. 2001), and 2) down-regulation of PRP genes in plants at specific stages of drought stress (Peng et al. 2015, Gujjar et al. 2018). It may be proposed that transcription of $P R P$ genes is discontinued or repressed by some unknown regulatory mechanism to substantiate the need of free proline in the cell during stress. However, the mechanisms underlying both of the above suggested events are yet to be discovered. Plenty of confusing questions are still left to be unravelled in the future, i.e.: Are PRPs degraded to liberate free proline or is their transcription blocked to substantiate a high content of free proline inside the cell during stress? After stress when cells do not require high content of proline, does transcription of PRP genes resume to normalize free proline content inside the cells? When proline content reaches toxic levels at any time during stress, does transcription of $P R P$ genes increase to consume free proline residues from cytosol? The future research should focus to unravel missing links between accumulation/depletion of proline and PRPs during and after stress. in pollen tube growth and early embryogenesis in Nicotiana tabacum. - Plant Cell Environ. 37: 499-511, 2014.

Deutch, C.E., Winicov, I.: Post-transcriptional regulation of a salt-inducible alfalfa gene encoding a putative chimeric proline-rich cell wall protein. - Plant mol. Biol. 27: 411-418, 1995.

Dvorakova, L., Cvrckova, F., Fischer, L.: Analysis of the hybrid proline-rich protein families from seven plant species suggests rapid diversification of their sequences and expression patterns. - BMC Genom. 8: 412, 2007.

Dvorakova, L., Srba, M., Opatrny, Z., Fischer, L.: Hybrid prolinerich proteins: novel players in plant cell elongation? - Ann. Bot. 109: 453-462, 2012.

Edstam, M.M., Viitanen, L., Salminen, T.A., Edqvist, J.: Evolutionary history of the non-specific lipid transfer proteins. - Mol. Plant 4: 947-964, 2011.

Esen, A., Bietz, J.A., Paulis, J.W., Wall, J.S.: Tandem repeats in the N-terminal sequence of a proline-rich protein from corn endosperm. - Nature 296: 678-679, 1982.

Fowler, T.J., Bernhardt, C., Tierney, M.L.: Characterization and expression of four proline-rich cell wall protein genes in Arabidopsis encoding two distinct subsets of multiple domain proteins. - Plant Physiol. 121: 1081-1091, 1999.

Francisco, S.M.K., Tierney, M.L.: Isolation and characterization of a proline-rich cell wall protein from soybean seedlings. Plant Physiol. 94: 1897-1902, 1990.

Gothandam, K.M., Nalini, E., Karthikeyan, S., Shin, J.S.: OsPRP3, a flower specific proline-rich protein of rice, determines extracellular matrix structure of floral organs and its overexpression confers cold-tolerance. - Plant mol. Biol. 72: 125-135, 2010.

Gujjar, R.S., Akhtar, M., Rai, A., Singh, M.: Expression analysis 
of drought induced genes in wild tomato line (Solanum habrochaites). - Curr. Sci. 107: 496-502, 2014.

Gujjar, R.S., Karkute, S.G., Rai, A., Singh, M., Singh, B.: Prolinerich proteins may regulate free cellular proline levels during drought stress in tomato. - Curr. Sci. 114: 915-920, 2018.

Hare, P., Cress, W.: Metabolic implications of stress induced proline accumulation in plants. - Plant Growth Regul. 21: 79102, 1997.

Hare, P.D., Cress, W.A., Van-Staden, J.: A regulatory role for proline metabolism in stimulating Arabidopsis thaliana seed germination. - Plant Growth Regul. 39: 41-50, 2003.

Hayat, S., Hayat, Q., Alyemeni, M.N., Wani, A.S., Pichtel, J., Ahmad, A.: Role of proline under changing environments: a review. - Plant Signal. Behav. 7: 1456-1466, 2012.

He, C.Y., Zhang, J.S., Chen, S.Y.: A soybean gene encoding a proline-rich protein is regulated by salicylic acid, an endogenous circadian rhythm and by various stresses. - Theor. appl. Genet. 104: 1125-1131, 2002.

Hong, J.C., Nagao, R.T., Key, J.L.: Developmentally regulated expression of soybean proline-rich cell wall protein genes. Plant Cell 1: 937-943, 1989.

Hong, J.C., Nagao, R.T., Key, J.L.: Characterization of a prolinerich cell wall protein gene family of soybean. A comparative analysis. - J. biol. Chem. 265: 2470-2475, 1990.

Hua, X.J., Van-de-Cotte, B., Van-Montagu, M., Verbruggen, N.: Developmental regulation of pyrroline-5-carboxylate reductase gene expression in Arabidopsis. - Plant Physiol. 114: 1215-1224, 1997.

José-Estanyol, M., Gomis-Rüth, F.X., Puigdomènech, P.: The eight-cysteine motif, a versatile structure in plant proteins. Plant Physiol. Biochem. 42: 355-365, 2004.

Kant, S., Kant, P., Raveh, E., Barak, S.: Evidence that differential gene expression between the halophyte Thellungiella halophila and Arabidopsis thaliana is responsible for higher levels of the compatible osmolyte proline and tight control of $\mathrm{Na}+$ uptake in T. halophila. - Plant Cell Environ. 29: 12201234, 2006.

Kishor, P.K., Hong, Z., Miao, G.H., Hu, C.A., Verma, D.P.: Overexpression of [delta]-pyrroline-5-carboxylate synthetase increases proline production and confers osmotolerance in transgenic plants. - Plant Physiol. 108: 1387-1394, 1995.

Kishor, P.K., Sangam, S., Amrutha, R.N., Laxmi, P.S., Naidu, K.R., Rao, K.R., Rao, S., Reddy, K.J., Theriappan, P., Sreenivasulu, N.: Regulation of proline biosynthesis, degradation, uptake and transport in higher plants: its implications in plant growth and abiotic stress tolerance. - Curr. Sci. 88: 424-438, 2005.

Lamport, D.T.A.: Isolation and partial characterization of hydroxyproline-rich glycopeptides obtained by enzymic degradation of primary cell walls. - Biochemistry 8: 11551163, 1969.

Li, B.C., Zhang, C., Chai, Q.X., Han, Y.Y., Wang, X.Y., Liu, M.X., Feng, H., Xu, Z.Q.: Plasmalemma localisation of double hybrid proline-rich protein 1 and its function in systemic acquired resistance of Arabidopsis thaliana. - Funct. Plant Biol. 41: 768-779, 2014.

Liu, A., Yu, Y., Li, R., Duan, X., Zhu, D., Sun, X., Duanmu, H., Zhu, Y.: A novel hybrid proline-rich type gene GsEARLI17 from Glycine soja participated in leaf cuticle synthesis and plant tolerance to salt and alkali stresses. - Plant Cell Tissue Organ Cult. 121: 633-646, 2015.

Maggio, A., Miyazaki, S., Veronese, P., Fujita, T., Ibeas, J.I., Damsz, B., Narasimhan, M.L., Hasegawa, P.M., Joly, R.J., Bressan, R.A.: Does proline accumulation play an active role in stress-induced growth reduction? - Plant J. 31: 699-712, 2002.

Mani, S., Van-de-Cotte, B., Van-Montagu, M., Verbruggen, N.:
Altered levels of proline dehydrogenase cause hypersensitivity to proline and its analogs in Arabidopsis. - Plant Physiol. 128 73-83, 2002.

Matysik, J., Bhalu, B., Mohanty, P.: Molecular mechanisms of quenching of reactive oxygen species by proline under stress in plants. - Curr. Sci. 82: 525-532, 2002.

Mellacheruvu, S., Tamirisa, S., Vudem, D.R., Khareedu, V.R.: Pigeonpea hybrid-proline-rich protein (CcHyPRP) confers biotic and abiotic stress tolerance in transgenic rice. - Front. Plant Sci. 6: 1167, 2016.

Menke, U., Renault, N., Mueller-Roeber, B.: StGCPRP, a potato gene strongly expressed in stomatal guard cells, defines a novel type of repetitive proline-rich proteins. - Plant Physiol. 122: 677-686, 2000.

Meringer, M.V., Villasuso, A.L., Margutti, M.P., Usorach, J., Pasquare, S.J., Giusto, N.M., Machado, E.E., Racagni, G.E.: Saline and osmotic stresses stimulate PLD/diacylglycerol kinase activities and increase the level of phosphatidic acid and proline in barley roots. - Environ. exp. Bot. 128: 69-78, 2016.

Mishra, S., Dubey, R.S.: Inhibition of ribonuclease and protease activities in arsenic exposed rice seedlings: role of proline as enzyme protectant. - J. Plant Physiol. 163: 927-936, 2006.

Nanjo, T., Fujita, M., Seki, M., Kato, T., Tabata, S., Shinozaki, K.: Toxicity of free proline revealed in an Arabidopsis T-DNAtagged mutant deficient in proline dehydrogenase. - Plant Cell Physiol. 44: 541-548, 2003.

Nieuwland, J., Feron, R., Huisman, B.A., Fasolino, A., Hilbers, C.W., Derksen, J., Mariani, C.: Lipid transfer proteins enhance cell wall extension in tobacco. - Plant Cell 17: 2009-2019, 2005.

Oono, Y., Seki, M., Nanjo, T., Narusaka, M., Fujita, M., Satoh, R., Satou, M., Sakurai, T., Ishida, J., Akiyama, K., Iida, K.: Monitoring expression profiles of Arabidopsis gene expression during rehydration process after dehydration using ca. 7000 full-length cDNA microarray. - Plant J. 34: 868-887, 2003.

Peng, T., Jia, M.M., Liu, J.H.: RNAi-based functional elucidation of PtrPRP, a gene encoding a hybrid proline rich protein, in cold tolerance of Poncirus trifoliata. - Front. Plant Sci. 6: 808, 2015.

Perez-Arellano, I., Carmona-Alvarez, F., Martinez, A.I., Rodriguez-Diaz, J., Cervera, J.: Pyrroline-5-carboxylate synthase and proline biosynthesis: from osmotolerance to rare metabolic disease. - Protein Science 19: 372-382, 2010.

Pitzschke, A., Xue, H., Persak, H., Datta, S., Seifert, G.J.: Post-translational modification and secretion of azelaic acid induced 1 (AZI1), a hybrid proline-rich protein from Arabidopsis. - Int. J. mol. Sci. 17: 85, 2016.

Priyanka, B., Sekhar, K., Reddy, V.D., Rao, K.V.: Expression of pigeonpea hybrid-proline-rich protein encoding gene (CcHyPRP) in yeast and Arabidopsis affords multiple abiotic stress tolerance. - Plant Biotechnol. J. 8: 76-87, 2010.

Qin, L.X., Zhang, D.J., Huang, G.Q., Li, L., Li, J., Gong, S.Y., Li, X.B., Xu, W.L.: Cotton GhHyPRP3 encoding a hybrid proline rich protein is stress inducible and its overexpression in Arabidopsis enhances germination under cold temperature and high salinity stress conditions. - Acta Physiol. Plant. 35: 1531-1542, 2013.

Rajendrakumar, C.S., Reddy, B.V., Reddy, A.R.: Proline-protein interactions: protection of structural and functional integrity of M4 lactate dehydrogenase. - Biochem. biophys. Res. Commun. 201: 957-963, 1994.

Rentsch, D., Hirner, B., Schmelzer, E., Frommer, W.B.: Salt stress-induced proline transporters and salt stress-repressed broad specificity amino acid permeases identified by 
suppression of a yeast amino acid permease-targeting mutant. - Plant Cell 8: 1437-1446, 1996

Ridge, I., Osborne, D.J.: Hydroxyproline and peroxidases in cell walls of Pisum sativum: regulation by ethylene. - J. exp. Bot. 21: 843-856, 1970.

Schwacke, R., Grallath, S., Breitkreuz, K.E., Stransky, E., Stransky, H., Frommer, W.B., Rentsch, D.: LeProT1, a transporter for proline, glycine betaine, and gamma-amino butyric acid in tomato pollen. - Plant Cell 11: 377-392, 1999.

Sharma, P., Dubey, R.S.: Modulation of nitrate reductase activity in rice seedlings under aluminium toxicity and water stress: role of osmolytes as enzyme protectant. - J. Plant Physiol. 162: 854-864, 2005.

Smirnoff, N., Cumbes, Q.J.: Hydroxyl radical scavenging activity of compatible solutes. - Phytochemistry 28: 1057-1060, 1989.

Stines, A.P., Naylor, D.J., Hoj, P.B., Heeswijack, R.: Proline accumulation in developing grapevine fruit occurs independently of changes in the levels of delta1-pyrroline5-carboxylate synthetase mRNA or protein. - Plant Physiol. 120: 923-923, 1999.

Szabados, L. Savoure, A.: Proline: a multifunctional amino acid. - Trends Plant Sci. 15: 89-97, 2010.

Thompson, J.F., Stewart, C.R., Morris, C.J.: Changes in amino acid content of excised leaves during incubation. I. The effect of water content of leaves and atmospheric oxygen level. -
Plant Physiol. 41: 1578-84, 1966.

Verbruggen, N., Hermans, C.: Proline accumulation in plants: a review. - Amino Acids 35: 753-759, 2008.

Verslues, P.E., Sharma, S.: Proline metabolism and its implications for plant-environment interaction. - Arabidopsis Book 8: e0140, 2010.

Wyatt, R.E., Nagao, R.T., Key, J.L.: Patterns of soybean prolinerich protein gene expression. - Plant Cell 4: 99-110, 1992.

Yamada, M., Morishita, H., Urano, K., Shiozaki, N., YamaguchiShinozaki, K., Shinozaki, K., Yoshiba, Y.: Effects of free proline accumulation in petunias under drought stress. - J. exp. Bot. 56: 1975-1981, 2005.

Yang, J., Yan, R., Roy, A., Xu, D., Poisson, J., Zhang, Y.: The I-TASSER suite: protein structure and function prediction. Nature Methods 12: 7-8, 2015.

Yeom, S.I., Seo, E., Oh, S.K., Kim, K.W., and Choi, D.: A common plant cell-wall protein HyPRP1 has dual roles as a positive regulator of cell death and a negative regulator of basal defense against pathogens. - Plant J. 69: 755-768, 2012.

Zhan, X., Wang, B., Li, H., Liu, R., Kalia, R.K., Zhu, J.K., Chinnusamy, V.: Arabidopsis proline-rich protein important for development and abiotic stress tolerance is involved in microRNA biogenesis. - Proc. nat. Acad. Sci. USA 109: 18198-18203, 2012. 